Revue d'histoire de l'Amérique française

DEVUE D.HISTOIRE DE L'AMÉRIQUE FRANÇAISE

\title{
Les plaideurs en Cour supérieure, 1880-1890 : classe, genre et juridicité durant la transition au capitalisme industriel
}

\section{Thierry Nootens}

Volume 68, numéro 1-2, été-automne 2014

URI : https://id.erudit.org/iderudit/1032018ar

DOI : https://doi.org/10.7202/1032018ar

Aller au sommaire du numéro

\section{Éditeur(s)}

Institut d'histoire de l'Amérique française

\section{ISSN}

0035-2357 (imprimé)

1492-1383 (numérique)

Découvrir la revue

\section{Citer cet article}

Nootens, T. (2014). Les plaideurs en Cour supérieure, 1880-1890 : classe, genre et juridicité durant la transition au capitalisme industriel. Revue d'histoire de l’Amérique française, 68(1-2), 25-56. https://doi.org/10.7202/1032018ar
Résumé de l'article

Nous avons examiné près de 700 poursuites entendues par la Cour supérieure dans le district de Trois-Rivières durant les années 1880. Plus d'une douzaine d'années se sont alors écoulées depuis la promulgation du Code civil du Bas-Canada de 1866. Au même moment, la transition au capitalisme industriel transforme les rapports de production. Deux questions générales ont guidé l'analyse : qu'est-ce que les dossiers de cette cour dévoilent quant aux enjeux sociojuridiques propres à une société régionale en transformation ? Cette documentation renvoie-t-elle à une phase particulière de l'histoire de la juridicisation et de la judiciarisation des rapports sociaux au Québec? 


\title{
Les plaideurs en Cour supérieure, I880-I890 : classe, genre et juridicité durant la transition au capitalisme industriel ${ }^{\mathrm{I}}$
}

\author{
Thierry NoOTENS \\ Département des sciences humaines \\ Université du Québec à Trois-Rivières \\ Centre interuniversitaire d'études québécoises (CIEQ)
}

RÉsumÉ • Nous avons examiné près de 700 poursuites entendues par la Cour supérieure dans le district de Trois-Rivières durant les années 1880. Plus d'une douzaine d'années se sont alors écoulées depuis la promulgation du Code civil du Bas-Canada de 1866. Au même moment, la transition au capitalisme industriel transforme les rapports de production. Deux questions générales ont guidé l'analyse: qu'est-ce que les dossiers de cette cour dévoilent quant aux enjeux sociojuridiques propres à une société régionale en transformation? Cette documentation renvoie-t-elle à une phase particulière de l'histoire de la juridicisation et de la judiciarisation des rapports sociaux au Québec?

ABSTRACT • The author analyzed 700 lawsuits heard by the Superior Court in the judicial district of Three-Rivers during the 1880s. A dozen years after the enactment of the Civil Code of Lower Canada (1866), industrial capitalism and market relations were transforming the economic and social fabric of Quebec. Two broad questions form the basis of this analysis: What do the judicial files of the Superior Court reveal about the social and legal stakes in a local society in transition? Do they portray a particular stage in the history of legalization and litigious nature of social relations in Quebec?

1. Cette recherche a bénéficié de l'appui du Conseil de recherches en sciences humaines du Canada. 


\section{INTRODUCTION}

Le droit civil et l'appareil chargé de le mettre en œuvre connaissent d'importants bouleversements au milieu du XIX ${ }^{\mathrm{e}}$ siècle. Certaines réformes viennent dégager les immeubles des rapports familiaux et communautaires dans lesquels ils se trouvaient enserrés à l'époque préindustrielle. Le douaire coutumier des femmes mariées, hypothèque légale sur les propres de leurs époux, est l'objet de restrictions à partir de $1841^{2}$. Le régime seigneurial, qui frappait les transactions foncières d'une taxe au profit des seigneurs (les lods et ventes), est aboli en $1854^{3}$. Le retrait lignager, droit de rachat d'un immeuble au bénéfice de la famille, disparaît en $1855^{4}$. Le rapport à l'argent se modifie aussi de manière substantielle. En 1850, on assiste à l'apparition du droit moderne des compagnies, ce qui facilite dès lors la mise en commun et la fructification de capitaux à grande échelle ${ }^{5}$. L'année 1858 , pour sa part, marque la fin du contrôle des taux d'intérêt dans les contrats et transactions de toutes sortes ${ }^{6}$.

D'autres réformes, proprement institutionnelles celles-là, mettent en place l’appareillage nécessaire à la vérification des transactions conclues par les particuliers et étendent l'emprise des tribunaux. Le système d'enregistrement créé par le Conseil spécial en 1841 va obvier à la difficulté, bien réelle, de connaître les charges affectant les immeubles ${ }^{7}$. En 1857, le nombre de districts judiciaires passe de 7 à $19^{8}$ afin de resserrer le quadrillage du territoire par la justice ${ }^{9}$. Simultanément, la pratique du droit se professionnalise. Le Barreau est incorporé en $1849^{10}$ et les programmes universitaires d'enseignement du droit se développent ${ }^{11}$. Qui plus est, la publication de périodiques de jurisprudence se régularise à partir des

2. Bettina Bradbury, «Debating Dower: Patriarchy, Capitalism and Widows' Rights in Lower Canada", dans Tamara Myers et al., dir., Power, Place and Identity: Historical Studies of Social and Legal Regulation in Quebec (Montréal, Montreal History Group, 1998): 55-78. Les propres sont les immeubles ne faisant pas partie de la communauté de biens.

3. Murray Greenwood, «Lower Canada (Quebec): Transformation of Civil Law, from Higher Morality to Autonomous Will, 1774-1866», Manitoba Law Journal, 23, 1-2 (1996) : 135, 160-161.

4. Ibid., 135-136, 145 et 161 .

5. Jean-Marie Fecteau, «Les "petites républiques" : les compagnies et la mise en place du droit corporatif moderne au Québec au milieu du XIX e siècle», Histoire sociale/Social History, 25, 49 (1992): 42.

6. M. Greenwood, «Lower Canada (Quebec)», loc. cit., 162.

7. Ibid., 139 et 158.

8. Pierre E. Audet, Les officiers de justice: des origines de la colonie jusqu'à nos jours (Montréal, Wilson et Lafleur, 1986), 63.

9. Brian Young, The Politics of Codification: The Lower Canadian Civil Code of 1866 (Montréal et Kingston, McGill-Queen's University Press, 1994), 100-101.

10. Ibid., 46.

11. Sylvio Normand, Le droit comme discipline universitaire : une histoire de la Faculté de droit de l'Université Laval (Québec, Les Presses de l’Université Laval, 2005), chap. 2 et 3. 
années 1850 , ce qui permettra de donner plus de prévisibilité au droit et à sa mise en œuvre par la judicature ${ }^{12}$.

Brian Young a bien montré en quoi les modifications apportées au droit civil sous l'Union (1841-1867) tendent à favoriser les échanges et la circulation des biens sur le marché, point focal du système capitaliste, cela au profit des classes possédantes et entrepreneuriales ${ }^{13}$. Le processus de codification, en particulier, est marqué par la valorisation du droit de propriété, de la liberté contractuelle et du respect des obligations ${ }^{14}$. Les commissaires abolissent la possibilité de rescision (annulation), pour cause de lésion, des contrats conclus entre majeurs, en tant qu'entorse à l'intégrité des contrats. Aussi, les clauses comminatoires (ou punitives) ne seront plus contrôlées par les tribunaux ${ }^{15}$. Et le consentement exprimé au contrat fera dorénavant de l'acquéreur le propriétaire de la chose, même avant que la tradition (transfert) de celle-ci n'ait lieu, ce qui accentue l'immédiateté des transactions nouées sur le marché ${ }^{16}$.

Si les réformes du milieu du XIX ${ }^{e}$ siècle de même que l'emprise de l'idéologie libérale sur la législation du Canada-Uni et le Code civil du BasCanada ont été bien documentées, le rôle joué par les tribunaux civils dans ce contexte demeure mal connu. En théorie, ce rôle est essentiel, puisque le marché ne peut se maintenir sans arbitrage des conflits, que suscite inévitablement son fonctionnement, et sans moyens permettant de forcer l'exécution des engagements et d'assurer le respect du droit de propriété. De plus, comme l'a signalé G. Blaine Baker, les chercheurs doivent encore établir «... the content of legal change wrought by the Civil Code of Lower Canada ${ }^{17} \ldots »$.

Exception faite des études basées sur l'exploitation des rapports de jurisprudence, les dossiers constitués à l'occasion de procès civils ont été

12. Eric Whan, Tamara Myers et Peter Gossage, «Stating the Case: Law Reporting in NineteenthCentury Quebec», dans Donald Fyson, Colin M. Coates et Kathryn Harvey, dir., Class, Gender and the Law in Eighteenth-and Nineteenth-Century Quebec: Sources and Perspectives (Montréal, Montreal History Group, 1993): 55-79.

13. Brian Young, "Positive Law, Positive State: Class Realignment and the Transformation of Lower Canada, 1815-1866", dans Allan Greer et Ian Radforth, dir., Colonial Leviathan: State Formation in MidNineteenth-Century Canada (Toronto, University of Toronto Press, 1992): 50-63.

14. Outre les travaux de Brian Young, voir également Sylvio Normand, «La codification de 1866 : contexte et impact», dans H. Patrick Glenn, dir., Droit québécois et droit français : communauté, autonomie, concordance (Cowansville, Les éditions Yvon Blais, 1993): 52.

15. M. Greenwood, «Lower Canada (Quebec)», loc. cit., 179-180.

16. B. Young, The Politics of Codification..., op. cit., 170 et ss.

17. G. Blaine Baker, «Introduction. Quebec and the Canadas, 1760 to 1867 : A Legal Historiography», dans G. Blaine Baker et Donald Fyson, dir., Essays in the History of Canadian Law, 11, Quebec and the Canadas (Toronto, University of Toronto Press, 2013): 12. 
mis à contribution afin d'examiner certains phénomènes spécifiques comme les conflits conjugaux ${ }^{18}$, l'expérience du veuvage ${ }^{19}$, diverses formes de déviance ${ }^{20}$, les disputes salariales ${ }^{21}$ ou, de manière originale, la culture juridique des avocats ${ }^{22}$. Seule Evelyn Kolish a examiné une part importante du contentieux de tribunaux en place durant la période ayant suivi la Conquête. Les dossiers de la Cour supérieure, pris en bloc, demeurent cependant terra incognita.

Kolish s'est penchée sur les causes entendues par la Cour des plaidoyers communs et la Cour du banc du Roi dans les districts de Montréal et Québec entre 1785 et 1825 , en accordant une attention particulière à la nature des litiges et à l'ethnicité des justiciables ${ }^{23}$. Elle a clairement établi la profusion des causes de recouvrement de dette et des affaires contractuelles. Kolish soutient que ces deux tribunaux n'interviennent que rarement dans les affaires privées. En témoignerait le petit nombre de causes relevant du droit familial au sens large (personnes et patrimoine) et de litiges immobiliers. Ainsi, l'appel aux tribunaux constituerait un dernier recours à l'époque préindustrielle en ce qui a trait à la régulation de la sphère privée. La force des réseaux familiaux, des réseaux communautaires et des structures de domination expliquerait la faible emprise de l'appareil judiciaire sur des populations rurales «... living in the relative isolation of family and community self-sufficiency alongside the commercial bustle in the cities ${ }^{24} »$.

Kolish clôt son étude sur une question: ce rapport particulier entre justice et société s'est-il transformé du fait de l'urbanisation, de l'industrialisation et de l'influence grandissante de l'économie urbaine sur les campagnes? À notre avis, il ne faut pas confondre sphère privée et ruralité. Néanmoins, la question de la disparition de l'autarcie relative des com-

18. Marie-Aimée Cliche, «Les procès en séparation de corps dans la région de Montréal, 1795-1879 », Revue d'histoire de l'Amérique française, 49, 1 (1995): 3-33.

19. Bettina Bradbury, Wife to Widow: Lives, Laws, and Politics in Nineteenth-Century Montreal (Vancouver, University of British Columbia Press, 2011), 502 p.

20. Thierry Nootens, Fous, prodigues et ivrognes : familles et déviance à Montréal au XIXe siècle (Montréal et Kingston, McGill-Queen's University Press, 2007), 308 p.

21. Grace Laing Hogg et Gwen Shulman, "Wage Disputes and the Courts in Montreal, 1816-1835 », dans D. Fyson, C. M. Coates et K. Harvey, dir., Class, Gender and the Law..., loc. cit., 127-143.

22. Jean-Philippe Garneau, «Une culture de l'amalgame au prétoire: les avocats de Québec et l'élaboration d'un langage juridique commun au tournant des $18^{\mathrm{e}}$ et $19^{\mathrm{e}}$ siècles ", The Canadian Historical Review, 88, 1 (2007): 113-148.

23. Evelyn Kolish, "Some Aspects of Civil Litigation in Lower Canada, 1785-1825: Towards the Use of Court Records for Canadian Social History", The Canadian Historical Review, 70, 3 (1989): 337-365. Notons que la Cour du banc du Roi exerçait aussi, alors, une juridiction civile de première instance.

24. Ibid., 365. 
munautés rurales face au commerce et aux interventions de la justice mérite d'être posée. Hors Québec, les analyses sérielles du contentieux des tribunaux civils sont tout aussi rares. Les travaux de Jacques Paul Couturier font exception. Couturier a mis en lumière les liens étroits unissant le monde des affaires et les activités des tribunaux dans une région excentrée du Nouveau-Brunswick à la fin du XIX ${ }^{\mathrm{e}}$ siècle. L'appareil judiciaire œuvre principalement, à cet endroit, au recouvrement de dettes et à l'arbitrage de causes contractuelles ${ }^{25}$.

Il faut évidemment s'attendre à ce que le dérèglement des transactions commerciales et les réclamations des créanciers, autrement dit les conflits relatifs aux choses pouvant être marchandées (les biens, l'argent), figurent en tête de liste des recours à la justice civile. Le droit des personnes et de la famille est moins lié aux aléas du marché, moins transactionnel, a fortiori dans les sociétés anciennes. Les résultats obtenus à partir des archives de tribunaux de la Nouvelle-France vont d'ailleurs dans le même sens ${ }^{26}$. Cette stabilité apparente de la composition du contentieux des tribunaux civils ne doit pas nous leurrer: une exploitation plus intensive des dossiers judiciaires est nécessaire. Bien entendu, une dette contractée à l'époque de la Nouvelle-France et une dette contractée à la fin du XIX ${ }^{e}$ siècle peuvent être issues de transactions bien différentes et mettre en scène des acteurs sociaux difficilement comparables.

L'histoire des tribunaux civils à l'époque préindustrielle ayant déjà été défrichée, nous avons ciblé une autre période, celle de la transition au capitalisme industriel. De plus, nous avons choisi d'examiner en bloc les activités d'un tribunal mis sur pied durant cette période charnière de l'histoire du Québec, en l'occurrence la Cour supérieure. Afin d'assurer la faisabilité de la démarche, seuls les procès entamés durant trois années (1880, 1885 et 1890) et dans un seul district (Trois-Rivières) ont été retenus.

Cette documentation s'avère un matériau de choix pour l'histoire économique et sociale. Il s'agit, à ce titre, de faire ressortir la signification de ces litiges en regard des divers modes de production, d'échange et de fructification de la valeur à cette époque, sans négliger les conflits interpersonnels qui se métamorphosent parfois en poursuites en bonne et due

25. Jacques Paul Couturier, "Courts and Business Activity in Late 19th Century New Brunswick: A View from the Case Files", Acadiensis, 26, 2 (1997): 81.

26. Colin M. Coates, "Community or Hierarchy?: Arguments Before the Seigneurial Court at Batiscan", dans D. Fyson, C. M. Coates et K. Harvey, dir., Class, Gender and the Law..., loc. cit., 84; John A. Dickinson, "La justice seigneuriale en Nouvelle-France: le cas de Notre-Dame-des-Anges», Revue d'histoire de l'Amérique française, 28, 3 (1974): 335 ; John A. Dickinson, Justice et justiciables: la procédure civile à la Prévôté de Québec, 1667-1759 (Québec, Les Presses de l’Université Laval, 1982), 123. 
forme. Aussi, ces procès ne sont pas nés dans un espace social neutre. Ils sont le produit de communautés traversées de rapports hiérarchiques et marquées par des inégalités substantielles. La condition sociale des demandeurs et défendeurs et la manière dont les groupes sociaux se confrontent dans l'enceinte du tribunal méritent par conséquent un examen attentif. Les archives de la Cour supérieure permettent en outre de réfléchir à une question de grande ampleur, celle de la juridicisation des rapports sociaux dans la province à cette époque. L'objectif, à cet égard, est de mettre en lumière la nature des liens de droit qui relient les uns aux autres les acteurs sociaux, l'éventail de ces liens ainsi que leur intensité. Ce matériel autorise également quelques conclusions quant à l'état de la judiciarisation du social à la fin du XIX ${ }^{e}$ siècle. Sur ce plan, c'est plutôt l'étendue du rôle et du pouvoir des tribunaux civils en matière de régulation des conflits qui doit être circonscrite ${ }^{27}$.

\section{LE TERRAIN D'ENQUÊTE}

La Cour supérieure est créée en $1849^{28}$ et sa juridiction s'étend à toute la province $^{29}$. Elle occupe dès lors une place de choix dans la hiérarchie des tribunaux civils et ses pouvoirs sont fort étendus ${ }^{30}$. Elle entend en première instance l'ensemble des affaires civiles, à l'exception des litiges relevant de la Cour de circuit et de l'Amirauté ${ }^{31}$. Ses décisions peuvent faire l'objet d'un appel à la Cour de révision et, subséquemment, à la Cour du banc de la Reine siégeant en appel en matières civiles ${ }^{32}$. Durant les années 1880 , elle est chargée d'emblée des réclamations de plus de $200 \$$, d'après la somme en jeu ou la valeur de la chose en litige, montant considérable pour l'époque. Les causes impliquant des sommes ou valeurs moindres échoient à la Cour de circuit. Les réclamations de $100 \$$ ou plus mais ne dépassant pas $200 \$$ constituent les causes appelables de la Cour de circuit, c'est-à-dire qu'elles peuvent être portées en appel devant la

27. Sur les concepts de juridicisation et judiciarisation, voir Jacques Commaille et Laurence Dumoulin, "Heurs et malheurs de la légalité dans les sociétés contemporaines. Une sociologie politique de la "judiciarisation" ", L'Année sociologique, 59, 1 (2009): 63-107.

28. Acte pour amender les lois relatives aux cours de juridiction civile en première instance, dans le Bas-Canada, 12 V. (1849), c. 38.

29. Les statuts refondus de la province de Québec, 1888, art. 2289.

30. Faute d'espace, nous ne ferons pas état de tous les pouvoirs de la cour, ni de toutes les nuances à apporter quant à ses rapports avec les autres tribunaux.

31. Pierre Basile Mignault, Code de procédure civile du Bas-Canada annoté (Montréal, J. M. Valois, 1891), art. 28.

32. Les statuts refondus de la province de Québec, 1888, art. 2295. 
Cour supérieure, à la différence des affaires de moins de $100 \$^{33}$. Les réclamations de moins de $100 \$$ découlant de droits immobiliers sont également susceptibles d'appel devant la Cour supérieure ${ }^{34}$. Les pouvoirs de la Cour supérieure en matière de brefs de capias ad respondendum font également en sorte qu'on ne peut pas seulement tenir compte du seuil monétaire de $200 \$$ au moment d'analyser le contentieux de ce tribunal. Des créanciers peuvent requérir l'arrestation de leurs débiteurs, même lorsque leur dette est moindre, en faisant valoir que ces derniers s'apprêteraient à les frauder en quittant le pays ou en dissimulant leurs avoirs ${ }^{35}$. Par conséquent, le corpus que nous avons constitué ne présente pas une unité parfaite sur le plan des montants ou de la valeur des biens en jeu. Sa cohérence relève plutôt de ce que les dossiers examinés constituent l'ensemble des litiges soumis en première instance à l'attention de la Cour supérieure dans le district de Trois-Rivières, durant les trois années ciblées.

Sont également de son ressort certains aspects délicats du droit des personnes, comme les tutelles et curatelles, tout comme les demandes de séparation de biens (dans le cas d'un mari éprouvant des difficultés financières) ou de séparation de corps (en raison d'un conflit conjugal exacerbé). De surcroît, ses pouvoirs de régulation institutionnelle sont considérables. Hormis la Cour du banc de la Reine, dans la province "... tous les tribunaux, magistrats et autres personnes, corps politiques et corporations [...] sont soumis au droit de surveillance et de réforme, aux ordres et au contrôle de la cour supérieure et de ses juges... », en vertu de ce que la loi prévoit ${ }^{36}$.

Durant la décennie 1880, le district judiciaire de Trois-Rivières couvre les comtés de Champlain, Maskinongé, Nicolet et Saint-Maurice, de même que la cité de Trois-Rivières ${ }^{37}$. Que le chercheur s'intéresse au contentieux ordinaire des tribunaux ou aux décisions qui firent jurisprudence, il ne peut faire fi des particularités de la société dont sont issus ces recours en justice. Cette contextualisation est une opération plus délicate qu'il n'y paraît. Les activités des tribunaux ne mettent pas le chercheur en communication directe avec une société ou ses «mentalités ${ }^{38}$ ». Ce sont les aléas des transactions conclues par les justiciables et les situations qu'ils

33. P. B. Mignault, Code de procédure civile du Bas-Canada annoté, op. cit., art. 1053 et 1054.

34. Ibid., art. 1054. Voir également l'article 1058.

35. Ibid., art. 808.

36. Les statuts refondus de la province de Québec, 1888, art. 2329.

37. Ibid., art. 70.

38. Pour un exemple de ce genre d'approche, voir J. A. Dickinson, Justice et justiciables..., op. cit., 99 et 117. 
jugent dignes ou susceptibles d'être corrigées par les tribunaux qui occupent ces derniers. La judiciarisation des rapports sociaux conflictuels est en bonne partie circonstancielle.

Mais les problèmes et disputes portés à l'attention de l'appareil judiciaire dépendent fondamentalement des rapports de production et de domination qui structurent l'existence des individus et organisations du milieu étudié. La région administrative de la Mauricie, qui correspond à un comté près au district judiciaire de Trois-Rivières des années 1880, est marquée à cette époque par la cohabitation de divers secteurs d'activité39 Si certaines productions agricoles des meilleurs terroirs sont commercialisées, on trouve des fronts pionniers plus pauvres aux abords du plateau laurentien. Les deux phares des industries mauriciennes sont le sciage du bois et la sidérurgie. L'exploitation des ressources forestières est dominée par un groupe restreint d'entrepreneurs anglophones disposant de larges concessions à l'intérieur des terres. La Mauricie n'a pas encore subi l'irruption du grand capital qui fera passer du bois scié aux pâtes et papiers l'usage principal de ses forêts. La sidérurgie régionale, pour sa part, entame son déclin précisément durant la décennie 1880. En fait, la conjoncture est mauvaise ces années-là : la crise économique ayant frappé l'Occident à partir de 1873 fait encore sentir ses effets. Cette société, dans son ensemble, est marquée par des inégalités sociales considérables. Les rapports de domination sont en outre fortement sexués eu égard, notamment, à l'infériorité juridique des femmes mariées. Enfin, honneur et réputation revêtent une importance particulière en raison d'une catholicité intense et du manque d'anonymat qui caractérise les rapports de sociabilité.

\section{LE CORPUS ET SON EXPLOITATION}

C'est de cette société régionale qu'est issu le contentieux examiné. La documentation à l'étude comprend toutes les poursuites entamées devant la Cour supérieure dans le district de Trois-Rivières en 1880, 1885 et 1890, soit un total de 689 dossiers. Ces dossiers sont conservés dans le fonds des matières civiles en général du greffe de Trois-Rivières de la Cour supérieure $^{40}$. Il peut y avoir plusieurs demandeurs ou défendeurs dans une

39. Les données qui suivent sont tirées de René Hardy et Normand Séguin, Histoire de la Mauricie (Sainte-Foy, Les Presses de l'Université Laval/Les Éditions de l'IQRC, 2004), 121 et ss.

40. Bibliothèque et Archives nationales du Québec (dorénavant BAnQ), Centre d'archives de la Mauricie et du Centre-du-Québec (dorénavant CMCQ), fonds des dossiers des matières civiles en général de la Cour supérieure, greffe de Trois-Rivières (fonds dorénavant désigné par sa cote, soit TP11 S3 SS2 SSS1). 
affaire, en vertu de l'objet des litiges. Du reste, un ou des demandeurs peuvent joindre dans une même poursuite des réclamations de type semblable à l'encontre d'un ou de plusieurs individus ${ }^{41}$. Ce faisant, une seule et même cause peut renvoyer à de multiples rapports sociojuridiques: prétentions de plusieurs justiciables en regard du même bien ou transactions multiples entre les mêmes parties. Ces affaires plus complexes ne constituent pas un obstacle. Elles permettent par exemple d'identifier les réseaux au sein desquels les habitants du district transigent, comme dans le cas de prêts impliquant à répétition les mêmes personnes.

Une cause devait avoir laissé des traces - si minces soient-elles - parmi les dossiers pour faire partie du corpus. Les procès ne s'étant pas terminés par un jugement en bonne et due forme, que ce soit en raison d'un désistement des demandeurs ou de la conclusion d'une entente hors cour, ont été retenus. Des dossiers semblaient incomplets, mais il a été possible d'obvier à cet état de chose en mettant systématiquement à contribution les entrées des plumitifs et, dans le cas des affaires ayant été menées à terme, les jugements consignés dans les registres de jugements.

Une base de données Filemaker Pro a été mise sur pied. Deux types de renseignements ont fait l'objet d'une attention spéciale: le fond du litige, présenté évidemment de manière variable par les demandeurs et les défendeurs (du moins par ceux de ces derniers qui se présentent en cour), de même que la profession des parties. Les causes ont été classées en dix catégories, en vertu de leur objet, alors que les demandeurs et défendeurs ont été répartis en dix groupes socioprofessionnels. Cette consolidation des données a pavé la voie à des analyses quantitatives réunissant groupes socioprofessionnels, statut dans les procédures et enjeux des poursuites. Les considérations qualitatives n’ont pas été négligées pour autant. Nous avons pris en compte les rapports sociaux spécifiques, microsociologiques même, dont font état et que cristallisent les poursuites. Une dispute testamentaire entre apparentés n'a pas la même résonance qu'un compte en souffrance chez un marchand de village, même si les montants en jeu sont semblables. Des chercheurs comme Viviana Zelizer ont depuis longtemps insisté pour que soient mis en lumière les effets sociaux des biens ${ }^{42}$. Parlons plutôt d'effets sociojuridiques: ces choses présentent divers degrés ou formes d'institutionnalisation du fait du droit, différentes juridicités qui méritent attention.

41. P. B. Mignault, Code de procédure civile du Bas-Canada annoté, op. cit., art. 15.

42. Viviana A. Zelizer, The Social Meaning of Money: Pin Money, Paychecks, Poor Relief, and Other Currencies (Princeton, Princeton University Press, 1997), 286 p. 
Le premier volet des résultats de cette enquête est consacré à la présentation des parties qui se font face en Cour supérieure dans le district de Trois-Rivières durant la décennie 1880. La situation désavantageuse des cultivateurs sur la scène judiciaire pointe en direction d'une persistance de l'exploitation de ce groupe social, comme c'était le cas à l'époque préindustrielle. Nous ferons ensuite état d'une autre situation sociojuridique difficile, celle des femmes. L'infériorité des femmes mariées et la mise à l'écart des femmes du monde des affaires pèsent très lourd sur la fréquence et les modalités de la présence féminine en justice. Nous examinerons pour finir le phénomène des apparitions multiples des mêmes plaideurs. Ces passages répétés devant le tribunal sont souvent le fruit de transactions conclues entre des justiciables de force inégale et des difficultés financières de certains d'entre eux.

\section{LE TRIBUNAL, SCÈNE DE LA CONFRONTATION DES GROUPES SOCIOPROFESSIONNELS?}

Les 689 dossiers à l'étude impliquent 1853 parties $^{43}$. Le nombre de parties est supérieur, du triple ou presque, au nombre de causes. De fait, les affaires comportant plusieurs demandeurs ou défendeurs ne sont pas rares. Qui plus est, les mêmes individus ou personnes morales peuvent poursuivre ou être poursuivis à de multiples reprises. Cela dit, les demandeurs et défendeurs ont été classés en dix catégories socioprofessionnelles en vertu des informations relevées dans les dossiers, sans réduire les réapparitions d'une même partie à une occurrence unique. Il s'agit, à ce stade, de voir globalement quel est le poids en justice des différents groupes composant la société du district de Trois-Rivières.

La grille utilisée (tableau 1) est fondée sur une répartition des individus en différents secteurs de production et d'échange de biens et services: agriculture, production artisanale, commerce, services professionnels, production manufacturière, etc. Quelques embûches durent être surmontées. Une lecture trop rapide des archives pourrait conduire à associer certains individus au mauvais secteur d'activité. Des «machinistes et forgerons » sont en fait des fabricants de pièces de moulin et non les ouvriers qualifiés que ces titres professionnels laissent supposer ${ }^{44}$. En outre, à certains justiciables sont associées deux occupations, comme dans le cas,

43. Ce sont les parties impliquées dans les procès au début de ceux-ci et dont font état les brefs d'assignation et les déclarations.

44. La vérification de l'appartenance socioprofessionnelle a été grandement facilitée par de multiples interrogations de la base de données Mauricie: base de données en histoire régionale, disponible en ligne au www.cieq.ca/ espacecieq.php?niv2=bases. 
assez fréquent, de ces «cultivateurs et commerçants». Cette pluriactivité est digne d'intérêt. Le fait que plusieurs individus tirent leur subsistance de deux secteurs d'activité peut être interprété comme un symptôme de la pénétration du marché et des occasions d'affaires au sein de cette société régionale, sans négliger, a contrario, la nécessité dans laquelle se trouvent certains habitants de travailler d'arrache-pied. Il a fallu faire des choix. Tous les individus dont l'énoncé des occupations inclut le titre de "cultivateur» ont été classés parmi les cultivateurs; le monde de la terre est leur ancrage. En d'autres cas, lorsque l'une des occupations mentionnées constitue un revenu d'appoint, l'occupation principale a déterminé le choix de catégorie. Enfin, les femmes mariées ne déclarent habituellement pas d'occupation à l'époque. Le cas échéant, elles ont été rangées dans le même groupe socioprofessionnel que celui de leur époux; les veuves dans celui de leur défunt mari; les héritières, dans celui du testateur les ayant gratifiées ${ }^{45}$. Ces différentes opérations de consolidation des données ont permis de réduire à près de $12 \%$ la proportion de cas où l'occupation des parties demeure un mystère ${ }^{46}$.

Les rôles joués dans les procès ont également été réduits, autant que possible, aux deux catégories générales de demandeur et défendeur. Dans leur immense majorité (près de $90 \%$ des cas), les parties sont désignées comme "demandeur» ou "défendeur». En matière de cession de biens et de faillite, les titres de "débiteur», de "débiteur cédant», de "failli» et d' "insolvable» ont été associés à un rôle de défendeur, comme c'est le mauvais état des affaires des personnes concernées qui suscite une procédure. Les quelques «mis en cause» furent de même inclus parmi les défendeurs, puisque les déclarations contiennent des reproches à leur égard. Les titres de "pétitionnaire » et "requérant» ont mené à une inclusion parmi les demandeurs; celui d' «intimé», parmi les défendeurs. Enfin, quelques rôles dans les procès ont été jugés indéterminés, vu leur irréductibilité à une position demanderesse ou défenderesse ${ }^{47}$.

Comme on pouvait s'y attendre, ce n'est pas là un reflet de la structure sociale du district. Se porter demandeur ou être contraint de se défendre en Cour supérieure implique, la plupart du temps, d'avoir été partie prenante de transactions assez substantielles ou de pouvoir faire valoir (ou

45. On peut certes être à la fois héritière et épouse. Par contre, les litiges successoraux impliquant des femmes font surtout état de la qualité d'héritière du de cujus.

46. Ce problème concerne plus souvent les défendeurs que les demandeurs. Le Code de procédure civile prévoit que le bref d'assignation doit inclure l'" occupation ou qualité » du demandeur alors que le nom du défendeur suffit. P. B. Mignault, Code de procédure civile du Bas-Canada annoté, op. cit., art. 49.

47. C'est le cas de syndics et de tiers-saisis. 


\section{Tableau I}

\section{Groupes socioprofessionnels et statut dans les procédures Cour supérieure, district de Trois-Rivières Dossiers des matières civiles en général, I 880- I 885 - 1890}

\begin{tabular}{|l|c|c|c|c|c|c|}
\hline \multirow{2}{*}{ Groupes socioprofessionnels $^{\mathbf{a}}$} & \multicolumn{2}{|c|}{ Demandeurs } & \multicolumn{2}{c|}{ Défendeurs } & \multicolumn{2}{c|}{ Total } \\
\cline { 2 - 7 } & $\mathrm{n}$ & $\%$ & $\mathrm{n}$ & $\%$ & $\mathrm{n}$ & $\%$ \\
\hline 1. Ouvriers et équivalents & 24 & 2,8 & 17 & 1,8 & 41 & 2,2 \\
\hline 2. Ouvriers spécialisés et artisans & 24 & 2,8 & 35 & 3,6 & 59 & 3,2 \\
\hline 3. Cultivateurs & 149 & 17,1 & 253 & 26,4 & 402 & 22,0 \\
\hline 4. Marchands et commerçants & 264 & 30,3 & 201 & 21,0 & 465 & 25,4 \\
\hline 5. Professions libérales & 93 & 10,7 & 76 & 7,9 & 169 & 9,2 \\
\hline 6. Cols blancs et équivalents & 50 & 5,7 & 50 & 5,2 & 100 & 5,5 \\
\hline 7. Industriels et manufacturiers & 96 & 11,0 & 66 & 6,9 & 162 & 8,9 \\
\hline 8. Personnes morales & 57 & 6,5 & 29 & 3,0 & 86 & 4,7 \\
\hline 9. Divers & 79 & 9,1 & 46 & 4,8 & 125 & 6,8 \\
\hline 10. Cas inconnus & 35 & 4,0 & 186 & 19,4 & 221 & 12,1 \\
\hline \multicolumn{1}{|c|}{ Total } & $\mathbf{8 7 1}$ & $\mathbf{1 0 0}$ & $\mathbf{9 5 9}$ & $\mathbf{1 0 0}$ & $\mathbf{1 8 3 0}$ & $\mathbf{1 0 0}$ \\
\hline
\end{tabular}

Source:BAnQ, CMCQ,TPII S3 SS2 SSSI, dossiers de I880, I885 et 1890.

a) Voici des exemples d'occupations propres aux catégories I à 9:

I. Ouvriers et équivalents: charretier, journalier, servante

2. Ouvriers spécialisés et artisans: briquetier, charpentier, cordonnier, ferblantier, fondeur, machiniste, mécanicien, menuisier, peintre, maçon

3. Cultivateurs: cultivateur

4. Marchands et commerçants: aubergiste, boulanger, boucher, colporteur, commerçant, marchand, marchand en gros, marchande publique, négociant, restaurateur

5. Professions libérales: avocat, médecin, magistrat, juge, notaire, pharmacien

6. Cols blancs et équivalents: agent d'assurances, arpenteur, commis, comptable, courtier, éditeur, maître de poste, institutrice, sténographe, teneur de livre, secrétaire-trésorier

7. Industriels et manufacturiers: propriétaire de fonderie, propriétaire de moulin, constructeur de chemin de fer, manufacturier

8. Personnes morales: banques, autres entreprises incorporées, municipalités

9. Divers: curé, prêtre, rentier, bourgeois, capitaine de navire, étudiant, organiste, gentilhomme, seigneur ou seigneuresse

b) Le nombre total de parties est légèrement inférieur aux I 853 parties des procès, en raison des 23 cas de statut indéterminé dans les procédures

se faire opposer) des droits pouvant conduire à des réclamations de plus de 200 \$. D’où la faible présence des ouvriers et ouvriers spécialisés. Les poursuites mettant en scène le menu peuple des villes et campagnes sont habituellement du ressort de la Cour de circuit ou de la Cour des com- 
missaires, tribunaux inférieurs ${ }^{48}$. Fait significatif, les ouvriers sont impliqués, dans le tiers des cas, dans des poursuites en dommages qui donnent lieu à des réclamations certes substantielles mais que le juge se charge de réduire à néant ou à peu de choses. Une épique chicane de voisinage opposant la famille d'un charretier à celle d'un journalier a été ponctuée de mots aigres-doux ("canaille», "diables», "voleurs», "ratatouille») et d'une accusation de vendre du lait impur ${ }^{49}$. L'affaire engendre une poursuite de 300 \$, rejetée par le magistrat.

Si les cultivateurs, individus dont la condition peut être variable, sont assez nombreux ( $22 \%$ des parties), leur présence dans le contentieux est près de trois fois inférieure, toutes proportions gardées, à leurs effectifs dans le district ${ }^{50}$. Le seuil monétaire de la juridiction du tribunal y est certainement pour beaucoup. Par contre, la justice civile n'est plus une réalité essentiellement urbaine comme à l'époque préindustrielle. Ses activités s'étendent bel et bien aux campagnes, même pour des litiges d'envergure $^{51}$. Le resserrement du quadrillage judiciaire de la province au milieu du XIX ${ }^{\mathrm{e}}$ siècle a porté fruit: le recours aux tribunaux en a été facilité, à n'en pas douter. Mais cette croissance institutionnelle n'aurait pas signifié grand-chose sans litiges à faire arbitrer et sans débiteurs forcés de rendre des comptes. La commercialisation de l'agriculture, l'essor du marché de la terre et le développement du marché de l'argent sont probablement en partie responsables de la représentation non négligeable des cultivateurs. Il aurait été étonnant de parvenir aux mêmes résultats si les communautés agraires et féodales du début du XIX ${ }^{e}$ siècle avaient été choisies comme terrain d'enquête. Le capital marchand avait peu d'emprise sur ces milieux caractérisés par la culture du blé, l'autosuffisance des familles et l'appropriation par le clergé et les seigneurs des surplus produits par les paysans ${ }^{52}$.

48. Sylvio Normand, «Justice civile et communauté rurale au Québec, 1880-1920», Les Cahiers de Droit, 25, 3 (1984): 605 .

49. BAnQ, CMCQ, TP11 S3 SS2 SSS1, 1885, nº 193, Gingras vs Livernoche.

50. Le monde agricole, à l'exclusion des villes et villages, rassemble près de $67 \%$ de la population de la Mauricie en 1891. R. Hardy et N. Séguin, Histoire de la Mauricie..., op. cit., 226, 227 et 259.

51. À l'époque de la Nouvelle-France, la justice civile entend essentiellement des causes nées en milieu urbain. J. A. Dickinson, Justice et justiciables..., op. cit., 142. Les analyses d'Evelyn Kolish ne comprennent pas de données relatives au statut socio-économique et au lieu de résidence des parties, ce qui rend toute comparaison impossible. Par contre, l'examen des litiges de la fin du XVIII siècle et du début du XIX ${ }^{\mathrm{e}}$ siècle montre, selon elle, que les tribunaux civils supérieurs n'ont alors qu'un rôle marginal dans la vie des populations rurales. E. Kolish, «Some Aspects of Civil Litigation in Lower Canada», loc. cit., 351 et 365.

52. Allan Greer, Habitants, marchands et seigneurs: la société rurale du bas Richelieu, 1740-1840 (Sillery, Septentrion, 2000), chap. 6 et 8 en particulier. 
Le groupe socioprofessionnel le mieux représenté est celui des marchands et commerçants, avec le quart des effectifs $(25,4 \%)$. Leur présence en justice serait jusqu’à dix fois supérieure à leur poids réel dans la population active de la région ${ }^{53}$. Bien implantés dans les villages et à TroisRivières, ils sont les maillons de chaînes de crédit aussi longues, complexes que fragiles ${ }^{54}$. Le report du paiement effectif des transactions, la possibilité d'une mauvaise récolte et les aléas de la conjoncture économique internationale contribuent à la volatilité du monde commercial au XIX ${ }^{\mathrm{e}}$ siècle $^{55}$. Sa régulation par les tribunaux revêt d'autant plus d'importance.

D’autres groupes socioprofessionnels sont manifestement surreprésentés dans le contentieux de la Cour supérieure, et de beaucoup: membres des professions libérales; industriels et manufacturiers; individus rangés dans la catégorie "divers", où figurent bon nombre de rentiers. Les services offerts par les premiers finissent par représenter une somme rondelette, en cas de procès compliqué. Des notaires et avocats, au demeurant, ne se contentent pas de leurs honoraires et transigent sur le marché des immeubles et de l'argent, à l'instar de certains rentiers. Les grosses affaires que brassent plusieurs industriels et manufacturiers en font de leur côté des plaideurs en Cour supérieure tout désignés.

La faible présence des personnes morales est remarquable. Les 86 apparitions de personnes morales concernent, en ordre décroissant: des banques (42), des municipalités (21), des entreprises manufacturières (10), des associations (4), des compagnies de chemin de fer (3), d'autres types d'entreprises (l'exploitante d'un pont, poursuivie deux fois, et une société de construction) (3), des compagnies d'assurances (2) et les syndics d'une paroisse (1). Les entreprises manufacturières d'envergure recensées sont toutes basées à l'extérieur de la région, sauf deux entités mises sur pied il y a peu (la Canada Iron Furnace Co. et la Laurentide Pulp Co.) et concernées chacune par un seul litige. À moins de supposer que les grandes entreprises soient demeurées à l'abri de poursuites ou de la nécessité de poursuivre durant la décennie 1880, le contentieux de la Cour supérieure témoigne de leur rôle encore modeste dans le district. La grande entre-

53. Pour un point de comparaison, voir Claude Bellavance, Yvan Rousseau et Jean Roy, Histoire du Centre-du-Québec (Québec, Les Presses de l’Université Laval, 2013), 243.

54. R. Hardy et N. Séguin, Histoire de la Mauricie..., op. cit., 328 et 329; Béatrice Craig, "Solder les comptes: les sources de crédits dans les magasins généraux ruraux de l'est canadien au milieu du XIX ${ }^{\mathrm{e}}$ siècle ", Journal of the Canadian Historical Association/Revue de la Société historique du Canada, 13, 1 (2002): 23-47.

55. Douglas McCalla, "An Introduction to the Nineteenth-Century Business World», dans Tom Traves, dir., Essays in Canadian Business History (Toronto, McClelland and Stewart, 1984), 18. 
prise industrielle incorporée n'a pas encore bouleversé la socio-économie locale. Le droit moderne des compagnies représente une innovation relativement récente dans les années $1880^{56}$ et des hommes d'affaires importants de la seconde moitié du XIX ${ }^{\mathrm{e}}$ siècle ne voient pas nécessairement d'intérêt à recourir à l'incorporation, lui préférant la forme plus flexible et moins régulée du partnership $p^{57}$. En outre, si notre échantillon recèle des individus impliqués dans des entreprises incorporées, ils poursuivent ou sont poursuivis à titre personnel. Le capitalisme et sa régulation judiciaire présentent un visage particulier dans les années 1880: celui de rapports interindividuels, de face-à-face. Nous aurons l'occasion d'y revenir.

L'appartenance socioprofessionnelle influence nettement le statut dans les procédures dans quelques cas. Les cultivateurs apparaissent à 149 reprises comme demandeurs, alors qu'ils sont défendeurs à 253 occasions. L'endettement chronique d'une frange importante des populations des campagnes y est pour beaucoup, puisque 95 de ces 253 rôles de défendeur découlent de poursuites pour des billets promissoires impayés. Ces effets de commerce, qui sont des promesses de paiement à échéance, peuvent avoir été signés à l'occasion de prêts ou de transactions diverses. Il s'est donc trouvé des individus pour transiger avec les ruraux, ce qui renforce la thèse d'une pénétration du marché en dehors des villes et agglomérations. De leur côté, les personnes morales agissent le plus souvent comme demanderesses. Les banques désireuses de recouvrer le paiement de tels billets promissoires y sont pour beaucoup. Enfin, dans la catégorie "divers» figurent maints individus désignés comme des «bourgeois» ou "rentiers», personnages en position de force en tant que prêteurs ou investisseurs sur le marché immobilier local.

La position en justice des différents groupes socioprofessionnels ne constitue qu'une mesure bien imparfaite des interactions sociales que les recours à la Cour supérieure viennent institutionnaliser. Si certains groupes sont plus souvent poursuivants que poursuivis, les membres d'une catégorie socioprofessionnelle donnée demandent-ils des comptes plus fréquemment à des concitoyens de condition différente? Le tableau 2 montre de quelle manière se font face les différents groupes socioprofessionnels. Notons que les occurrences relevées constituent des liens noués en justice. Si, par exemple, un demandeur de profession libérale a

56. J.-M. Fecteau, «Les "petites républiques”...», loc. cit.

57. C. Ian Kyer, «Gooderham \& Worts: A Case Study in Business Organization in Nineteenth-Century Ontario ", dans G. Blaine Baker et Jim Phillips, dir., Essays in the History of Canadian Law, 8, In Honour of R. C. B. Risk (Toronto, University of Toronto Press, 1999): 335-357. 
Tableau 2

Confrontation des groupes socioprofessionnels en vertu du statut dans les procédures

\section{Cour supérieure, district de Trois-Rivières}

Dossiers des matières civiles en général, I 880 - I 885 - 1890

\begin{tabular}{|l|c|c|c|c|c|c|c|c|c|c|c|}
\hline \multirow{2}{*}{\begin{tabular}{|c|c|c|c|c|c|c|} 
Demandeurs : \\
groupes socioprofessionnels
\end{tabular}} & \multicolumn{7}{|c|}{ Défendeurs : groupes socioprofessionnels } & \multirow{2}{*}{ Total } \\
\cline { 2 - 13 } & $1^{\mathrm{a}}$ & 2 & 3 & 4 & 5 & 6 & 7 & 8 & 9 & 10 & \\
\hline 1. Ouvriers et équivalents & 6 & 8 & 2 & 2 & 0 & 0 & 0 & 1 & 0 & 8 & 27 \\
\hline $\begin{array}{l}\text { 2. Ouvriers spécialisés } \\
\text { et artisans }\end{array}$ & 2 & 6 & 7 & 2 & 0 & 0 & 0 & 4 & 1 & 5 & 27 \\
\hline 3. Cultivateurs & 6 & 6 & 96 & 24 & 6 & 7 & 8 & 7 & 9 & 34 & 203 \\
\hline 4. Marchands et commerçants & 2 & 8 & 73 & 127 & 10 & 9 & 12 & 8 & 13 & 85 & 347 \\
\hline 5. Professions libérales & 1 & 4 & 38 & 16 & 15 & 11 & 6 & 3 & 5 & 22 & 121 \\
\hline 6. Cols blancs et équivalents & 0 & 3 & 27 & 5 & 8 & 5 & 1 & 5 & 4 & 11 & 69 \\
\hline 7. Industriels et manufacturiers & 0 & 0 & 17 & 28 & 6 & 1 & 28 & 2 & 10 & 26 & 118 \\
\hline 8. Personnes morales & 0 & 2 & 17 & 31 & 19 & 9 & 16 & 1 & 4 & 20 & 119 \\
\hline 9. Divers & 2 & 3 & 35 & 15 & 12 & 8 & 10 & 1 & 7 & 13 & 106 \\
\hline 10. Cas inconnus & 0 & 0 & 12 & 6 & 4 & 0 & 0 & 2 & 5 & 10 & 39 \\
\hline Total & 19 & 40 & 324 & 256 & 80 & 50 & 81 & 34 & 58 & 234 & 1176 \\
\hline
\end{tabular}

Source: BAnQ, CMCQ,TPII S3 SS2 SSSI, dossiers de I880, 1885 et 1890 .

a) Les chiffres placés en tête des colonnes renvoient aux groupes socioprofessionnels correspondants.

poursuivi simultanément un ouvrier et un marchand, dans une même cause, deux occurrences distinctes ont été comptabilisées.

Pour ce qui est des interactions judiciaires à l'intérieur des mêmes groupes, on constate que les justiciables des catégories les moins favorisées (catégories 1 à 3) ont tendance à se poursuivre entre eux. Aucun ouvrier ou ouvrier spécialisé ne procède contre un industriel. La remarque vaut aussi pour les marchands et commerçants qui actionnent très souvent leurs semblables, à l'instar des industriels et manufacturiers. Sur le plan des rencontres en justice de groupes sociaux distincts, la situation des cultivateurs et des marchands retient l'attention. Les membres des professions libérales et les cols blancs demandent fréquemment des comptes aux premiers, tandis que les seconds doivent répondre de bon nombre de réclamations présentées par des industriels et des personnes morales. En somme, les campagnards, sous-représentés dans le contentieux de la Cour supérieure, et les gens du négoce, qui y sont surreprésentés, polarisent les 
poursuites. Tant celles issues du même milieu socioprofessionnel que celles en provenance d'autres milieux.

Tout compte fait, l'analyse quantitative de la condition sociale et du statut des parties se prête mal à une lecture trop marxisante. La Cour supérieure n'est pas le lieu où s'expriment de manière crue les rapports socio-économiques inégalitaires, bien réels, qui marquent la vie des populations du district durant la seconde moitié du XIX ${ }^{\mathrm{e}}$ siècle. Les conflits de travail sont plus significatifs. En 1880, Le Journal des Trois-Rivières avertit les travailleurs des fonderies de la ville de ne pas se rendre aux arguments de représentants de «l'association des ouvriers mouleurs de Montréal» venus leur faire prendre "... l'engagement de ne travailler qu'à raison de $\$ 1,50$ par jour». Au dire du journal, ce sont des sbires de ces "associations communardes " qui suscitent grèves et violences ${ }^{58}$. On aura pris acte, néanmoins, de la position de faiblesse des cultivateurs, donnée qui traduit indirectement l'exploitation dont ils sont l'objet. Mais ce qui nourrit le contentieux du tribunal, ce sont les aléas des transactions conclues par les justiciables et les conflits de tout acabit qui ponctuent leur existence. Ce faisant, l'appartenance de classe revêt moins d'importance que les intérêts particuliers. Qu'il s'agisse de poursuivre des apparentés au sujet d'un héritage ou de faire rendre gorge à un partenaire d'affaires qui ne rembourse pas ses dettes, cette logique prévaudra. Comme on le verra, c'est plutôt en plongeant dans le détail de dossiers spécifiques que l'on peut mettre en lumière des situations très nettes de domination subie par des habitants du district aux mains d'individus mieux nantis, cela à l'échelle des villages, des paroisses ou des microréseaux au sein desquels naissent et circulent les créances.

\section{GENRE ET JUSTICE: LA PRÉSENCE DES FEMMES}

Il est par contre une forme d'inégalité qui transcende les hiérarchies sociales et qu'un survol de l'identité des demandeurs et défendeurs met d'emblée en évidence, celle du genre. Si, de manière générale, les distinctions de classe ne s'accompagnent pas d'un statut juridique particulier - ce qui répugnerait à l'idéologie libérale, dont l'une des pierres d'assise est l'égalité formelle des citoyens - le droit civil institutionnalise des rapports lourdement inégalitaires entre les sexes. Jusque tard au XXe siècle, c'est le mariage qui contraint plus particulièrement les femmes, les veuves et célibataires jouissant d'une autonomie à tout le moins formelle. La 
condition juridique des femmes mariées ayant été analysée en maints endroits, rappelons seulement leur devoir d'obéissance et leur incapacité générale à contracter ${ }^{59}$. Seul un contrat de mariage en séparation de biens peut leur offrir une certaine latitude, tout comme l'état de marchande publique ou un jugement de séparation de $\operatorname{corps}^{60}$. Leur accès à la justice civile est on ne peut plus contrôlé : peu importe leur situation, les épouses ne peuvent en dehors de cas très précis ester en justice sans assistance ou autorisation maritale, autorisation à laquelle peut suppléer un juge ${ }^{61}$.

L'infériorité juridique des épouses et leur incapacité à ester en justice se répercutent clairement sur le contentieux de la Cour supérieure. Notre échantillon de 689 litiges met en scène 204 demanderesses et défenderesses contre 1540 demandeurs et défendeurs ${ }^{62}$. Cette disproportion est symptomatique de l'impossibilité dans laquelle se trouvent bon nombre de femmes de conclure des transactions d'envergure. Leur statut dans les procédures est tout aussi significatif. Les femmes agissent à 125 reprises comme demanderesses et à 79 reprises en tant que défenderesses. Leurs droits et avoirs sont probablement menacés plus souvent qu'à leur tour, du fait de leur condition subalterne. Les tribunaux civils, s'ils sont chargés de mettre en ouvre un droit profondément patriarcal, constituent des rouages importants de la régulation de l'infériorité des femmes au XIX siècle. Dit autrement, leur sort n'est pas livré à la seule brutalité institutionnalisée des rapports familiaux et des pratiques religieuses qui contraignent leur existence. Le positionnement judiciaire des hommes est différent: ils sont 689 fois demandeurs et défendeurs à 851 occasions.

Le tableau 3 situe les plaideuses au sein des différents milieux socioprofessionnels. Les résultats obtenus sont quelque peu trompeurs, comme les femmes sans occupation apparente en dehors du foyer ont été associées

59. Code civil du Bas-Canada (dorénavant $C C B C$ ), art. 174 et 177.

60. L'épouse séparée de biens jouit de la simple administration de ses avoirs, mais elle ne peut conclure elle-même une transaction majeure comme la vente d'un immeuble (CCBC, art. 177). Sous le régime de la communauté de biens, l'homme est maître des décisions relatives à ce patrimoine durant la vie commune (CCBC, art. 1292). Une marchande publique peut sans autorisation maritale «... s'obliger pour ce qui concerne son négoce...", mais elle doit avoir été autorisée expressément ou tacitement pour agir en tant que telle (CCBC, art. 179). L'épouse séparée de corps est en mesure de contracter seule, hormis pour aliéner un immeuble, auquel cas le juge devra l'autoriser (CCBC, art. 210).

61. $C C B C$, art. 176 et 178. Les épouses séparées de biens peuvent par contre plaider dans les cas de simple administration, par exemple dans le but de percevoir les revenus produits par un immeuble leur appartenant. Une épouse séparée de corps est capable d'ester en jugement, mais un juge devra l'autoriser pour les «... poursuites tendant à l'aliénation de ses immeubles...». CCBC, art. 210.

62. Rappelons qu'il s'agit de rôles joués dans les procédures et non d'individus distincts, car des justiciables apparaissent plus d'une fois dans le contentieux. Le total de parties «sexuées» est de 1744. Des 1830 rôles de demandeurs et défendeurs - les statuts indéterminés ayant été retranchés - nous devons soustraire les 86 apparitions de personnes morales, d'où le total de 1744 . 
Tableau 3

Les femmes: groupes socioprofessionnels et statut dans les procédures

Cour supérieure, district de Trois-Rivières Dossiers des matières civiles en général, I 880 - I 885 - 1890

\begin{tabular}{|l|c|c|c|c|c|c|}
\hline \multirow{2}{*}{ Groupes socioprofessionnels } & \multicolumn{2}{|c|}{ Demanderesses } & \multicolumn{2}{|c|}{ Défenderesses } & \multicolumn{2}{|c|}{ Total } \\
\cline { 2 - 8 } & $\mathrm{n}$ & $\%$ & $\mathrm{n}$ & $\%$ & $\mathrm{n}$ & $\%$ \\
\hline 1. Ouvriers et équivalents & 8 & 6,4 & 4 & 5,1 & 12 & 5,9 \\
\hline 2. Ouvriers spécialisés et artisans & 2 & 1,6 & 5 & 6,3 & 7 & 3,4 \\
\hline 3. Cultivateurs & 19 & 15,2 & 17 & 21,5 & 36 & 17,6 \\
\hline 4. Marchands et commerçants & 30 & 24,0 & 9 & 11,4 & 39 & 19,1 \\
\hline 5. Professions libérales & 18 & 14,4 & 15 & 19,0 & 33 & 16,2 \\
\hline 6. Cols blancs et équivalents & 7 & 5,6 & 3 & 3,8 & 10 & 4,9 \\
\hline 7. Industriels et manufacturiers & 7 & 5,6 & 4 & 5,1 & 11 & 5,4 \\
\hline 8. Personnes morales & - & - & - & - & - & - \\
\hline 9. Divers & 12 & 9,6 & 5 & 6,3 & 17 & 8,3 \\
\hline 10. Cas inconnus & 22 & 17,6 & 17 & 21,5 & 39 & 19,1 \\
\hline Total & $\mathbf{1 2 5}$ & $\mathbf{1 0 0}$ & $\mathbf{7 9}$ & $\mathbf{1 0 0}$ & $\mathbf{2 0 4}$ & $\mathbf{1 0 0}$ \\
\hline
\end{tabular}

Source: BAnQ, CMCQ,TPII S3 SS2 SSSI, dossiers de 1880, I885 et I890.

au secteur d'activité d'hommes de leur entourage ${ }^{63}$. Dans près de $20 \%$ des cas, ni données occupationnelles ni informations sur le milieu familial ne sont disponibles. En regard de la répartition générale des demandeurs et défendeurs en différents groupes socioprofessionnels, la condition sociale des femmes ne présente pas de différence significative, hormis une représentation plus importante des ménages où le chef de famille exerce ou a exercé une profession libérale. Le monde de la terre et le négoce dominent tout autant.

Alors que la désignation des justiciables mâles dans les procédures inclut la plupart du temps (à près de $90 \%$ ) la mention d'une occupation, les données qui complètent la désignation des femmes dans les déclarations et brefs d'assignation sont de trois types: statut matrimonial, statut patrimonial ou occupation proprement dite. Ces diverses qualités peuvent

63. Voir plus haut. Évidemment, aucune femme n'exerce une profession libérale à l'époque. Il s'agit ici d'inscrire les plaideuses au sein des rapports de classe, tels que les recours en justice en témoignent. La femme d'un industriel n'est pas du même rang que l'épouse d'un cultivateur, même en l'absence de métier en propre. 
d'ailleurs se combiner. Le statut matrimonial est fréquemment spécifié, comme il détermine pour une bonne part la capacité de poursuivre ou d'être poursuivie. Le fait d'être séparée de biens (60 occurrences) ou veuve (53) favorise certainement la présence des femmes en Cour supérieure, en regard des plaideuses dont on mentionne seulement la qualité d'épouse d'un individu (63). Nous avons aussi repéré une "fille mineure», Amanda Ringuette, une servante qui poursuit un habitant de Sainte-Anne-deYamachiche pour 250 \$, sans que nous sachions pour quel motif. Un tuteur l'a-t-elle accompagnée dans sa démarche ${ }^{64}$ ? Dans 27 cas, le statut matrimonial demeure inconnu.

Pour ce qui est des rôles patrimoniaux, huit mentions de tutrices ont été relevées. En charge de la personne et des biens de mineurs, les tutrices sont toutefois soumises à la supervision des conseils de famille et de l'appareil judiciaire lorsque des décisions importantes doivent être prises, comme dans le cas de la vente d'immeubles. Une seule femme cumule les rôles de tutrice, d'exécutrice testamentaire et de légataire fiduciaire de son défunt mari. Il s'agit de Caroline Arlène Pothier, veuve de Petrus Odilon Désilets désigné de son vivant comme bourgeois ou rentier. C'est dire le rôle central qui est le sien auprès de son fils Lucien, un des légataires universels. Par contre, elle a un temps partagé ses fonctions d'exécutrice testamentaire et de légataire fiduciaire avec cinq hommes, dont deux notaires dont le patronyme est Désilets. L'autonomie offerte par le veuvage est manifestement relative dans son cas, du moins en ce qui a trait à la gestion des actifs familiaux ${ }^{65}$. Pour le reste, on ne compte qu'une curatrice. Il y a peu de femmes, enfin, auxquelles sont apposés des titres successoraux: cinq héritières, six légataires et une usufruitière. La gestion des tutrices et curatrices ainsi que les transmissions de biens impliquant des femmes ne semblent pas marquées par une litigiosité très vive. L'encadrement social et judiciaire des tutrices et curatrices est substantiel. Les règles du Code civil et le recours au savoir des notaires font aussi en sorte, peut-on croire, que les successions se déroulent la plupart du temps de manière assez prévisible, sans trop de mauvaises surprises.

Les femmes qui déclarent ou auxquelles on appose une occupation précise sont rarissimes: une aubergiste, une institutrice, une restauratrice et une servante. Olivine Gauthier, aubergiste de Trois-Rivières et veuve d'Édouard Dupont, est poursuivie en 1890 par deux individus associés en

64. BAnQ, CMCQ, TP11 S3 SS2 SSS1, 1880, $\mathrm{n}^{\circ} 102$, Ringuette $v s$ Desaulniers.

65. BAnQ, CMCQ, TP11 S3 SS2 SSS1, 1880, ${ }^{\circ} 60$, Saint-Louis ${ }^{v s}$ Désilets; 1885, ${ }^{\circ}{ }^{93}$, Désilets vs Desaulniers. 
affaires, Nazaire et Joseph-Antoine Gagnon. Elle aurait signé deux billets promissoires à leur ordre en 1885 ; le capital et les intérêts s'élèvent cinq ans plus tard à près de $250 \$$. Alors que bien des individus poursuivis pour des billets promissoires impayés ne comparaissent pas, Olivine se défend. Les billets sont faux, dit-elle; elle sait lire et signe elle-même les obligations qu'elle contracte; les demandeurs ont pressé son mari pour que ce dernier signe les billets à sa place. Il s'agirait donc d'une dette de son mari et elle a renoncé à la communauté de biens qui les unissait de son vivant. Les Gagnon ont-ils cherché à garantir une dette de la communauté pour des marchandises impayées, avant le décès du mari d'Olivine, en faisant de celle-ci leur débitrice personnelle? Le cas échéant, cette stratégie échoue. Le juge renvoie la poursuite ${ }^{66}$.

Si le monde du petit commerce, où œuvrent bien des femmes, échappe en bonne partie au regard de la Cour supérieure, on compte tout de même 13 apparitions de marchandes publiques dans le corpus ${ }^{67}$. Dix femmes distinctes sont concernées. Ce nombre est bien faible en regard de la foule de commerçants mâles qui se pressent aux portes du tribunal. Mais ces procès sont dignes d'intérêt du point de vue des rapports entre femmes et monde des affaires au XIX ${ }^{\mathrm{e}}$ siècle.

Theresa Maria Sharples semble en difficulté en 1885. Elle est poursuivie à quatre reprises, notamment en qualité de "manufacturière et marchande publique, société E. Bradley et Co.». Son mari, Earnshaw Bradley, ingénieur civil, est toujours en vie et le couple est séparé de biens. Le régime matrimonial des marchandes publiques a de l'importance: il détermine si l'homme sera responsable des obligations contractées par sa femme, ce qui est le cas si le couple est en communauté de biens. Une marchande publique séparée de biens peut ainsi mettre un époux à l'abri, s'il conduit les opérations sous couvert de sa femme ${ }^{68}$. Bradley est (ou a été) le partenaire d'hommes d'affaires du Massachusetts impliqués dans le commerce d'écorce de pruche, secteur d'activité de la même E. Bradley et Co. ${ }^{69}$. Pour quelle raison Theresa se retrouve-t-elle marchande publique? Envisageaitelle cette vocation au moment de son mariage? 
Toujours est-il qu'en 1885 elle est poursuivie deux fois par les frères Gault, les rois pancanadiens de l'industrie textile, au sujet de deux billets promissoires qu'elle a signés elle-même et dont ils se sont retrouvés propriétaires; par des marchands de Trois-Rivières, pour un compte de marchandises; par un «bourgeois » d'Acton Vale à propos d'un billet promissoire signé par un tiers à l'ordre de la E. Bradley et Co. ${ }^{70}$. Manifestement, son statut de marchande publique, qui lui permet de «... s'obliger pour ce qui concerne son négoce...» sans autorisation maritale ${ }^{71}$, l’a dotée d'une autonomie considérable. Par contre, si son arrivée à la tête des entreprises de son mari, qu'il s'agisse d'un simulacre ou d'une réelle passation des pouvoirs, était destinée à obvier aux difficultés financières de celui-ci ${ }^{72}$, la situation du couple ne semble pas se redresser. Dans tous les cas, elle est condamnée à payer les sommes réclamées.

Dans le cadre du procès intenté par l'homme d'Acton Vale, un certain Van, elle est poursuivie conjointement avec un avocat de Trois-Rivières, Martin Honan. Celui-ci a signé un billet promissoire de près de $500 \$$ à l'ordre de la E. Bradley et Co. et s'est ainsi engagé, apparemment, à payer cette somme à l'échéance prévue, soit quatre mois plus tard. Honan affirme pour sa défense qu'il a fait ce geste pour prêter son crédit à Theresa - qui donc n'en avait plus tellement peut-on croire - et que cette dernière avait promis de solder le billet à échéance. Ce qu'elle n'a pas fait. Elle s'est probablement servie de cet effet de commerce pour obtenir des fonds ou solder des comptes auprès de $\mathrm{M}$. Van, à qui elle a transporté le billet, engageant du même coup la responsabilité d'Honan et la sienne envers $\operatorname{Van}^{73}$.

Trois autres marchandes publiques sont associées, en titre, à une société au nom de leur mari. Ce dernier vit encore et elles se sont toutes mariées sous le régime de la séparation de biens. Le fait qu'à des marchandes publiques soit associée une entreprise au nom de leur mari ne permet pas de conclure à un rôle de façade. À l'inverse, une firme au nom de l'épouse ne garantit pas une direction effective de sa part ${ }^{74}$. En principe, le statut de marchande publique la rend autonome en ce qui concerne les opérations commerciales véritablement conduites par elle-même et non en ce qui a trait à celles de son époux. Il existe au moins un cas apparent d'ins-

70. BAnQ, CMCQ, TP11 S3 SS2 SSS1, 1885, $\mathrm{n}^{\circ}$ 90, Gault vs Sharples; 1885, ${ }^{\circ}$ 167, Gagnon vs Sharples; $1885, \mathrm{n}^{\circ}$ 198, Van $v$ S Sharples; $1885, \mathrm{n}^{\circ}$ 206, Gault vs Sharples.

71. $C C B C$, art. 179.

72. Le cas existe. B. Young, The Politics of Codification..., op. cit., 155.

73. BAnQ, CMCQ, TP11 S3 SS2 SSS1, 1885, n ${ }^{\circ} 198$, Van vs Sharples.

74. B. Young, "Getting Around Legal Incapacity... », loc. cit., 9 et 11. 
trumentalisation de ce statut. Le 8 avril 1890, Célina Duval s'adresse à la justice afin que, dorénavant, elle soit séparée de biens de son mari, le marchand François-Xavier Sarrasin de Trois-Rivières. Ce changement de régime matrimonial requiert que les droits de l'épouse dans la communauté soient menacés par le mauvais état des affaires du chef de ménage ${ }^{75}$. Célina n'est pas alors désignée comme marchande publique. Le 17 mai suivant, le juge accède à sa requête. Il lui donne acte de sa renonciation à la communauté de biens qui l'a liée à Sarrasin et lui permet de jouir de ses biens et de les administrer elle-même ${ }^{76}$. Or, le 3 septembre 1890, elle poursuit en son nom propre un cultivateur pour la somme de $105 \$$ en tant que «marchande publique, société F.X. Sarrasin et Cie $»^{77}$. Les entreprises de Sarrasin n'ayant pas laissé de traces dans la presse régionale, on peut croire que son commerce est de taille modeste. La collaboration des femmes mariées à la bonne marche des petites boutiques est certes monnaie courante. Mais de quels actifs Célina a-t-elle disposé pour maintenir ce commerce en vie? De quels avoirs put-elle jouir à partir de l'octroi de la séparation de biens par le juge, eu égard à sa renonciation à la communauté?

S'il est des marchandes publiques que l'on peut qualifier de circonstancielles, d'autres ont probablement dirigé leur propre commerce. C'est le cas d'une veuve du village de La Rochelle et d'une célibataire de TroisRivières. Elles sont toutes deux visées par des demandes de cession de biens présentées par des marchands de Montréal, des grossistes, ce qui ferait d'elles des détaillantes sur le marché régional ${ }^{78}$.

\section{LES DEMANDEURS ET DÉFENDEURS RÉCURRENTS}

Le contentieux de la Cour supérieure présente donc un visage particulier. La condition sociale et le sexe des plaideurs le structurent de manière importante. Le seuil monétaire de la juridiction du tribunal y est pour beaucoup, tout comme l'incapacité des femmes mariées. La besogne abattue par les tribunaux relève en revanche d'une «demande sociale» de régulation judiciaire. Mais avoir des droits à faire valoir ne suffit pas, quoique le bien-fondé d'une réclamation soit essentiel, à l'instar de la preuve qui pourra l'étayer. Des pressions ou négociations informelles ont

75. CCBC, art. 1311.

76. BAnQ, CMCQ, TP11 S3 SS2 SSS1, 1890, n² 229, Duval vs Sarrasin.

77. BAnQ, CMCQ, TP11 S3 SS2 SSS1, 1890, n 244, Duval vs Belleville.

78. BAnQ, CMCQ, TP11 S3 SS2 SSS1, 1890, n 557, Adams vs Daignault; 1890, $\mathrm{n}^{\circ}$ 598, Paterson vs Larivière. 
peut-être achoppé ; les ressources nécessaires pour faire face à un procès et en assumer les risques (comme une condamnation aux dépens) sont fort inégalement réparties; l'espoir d'obtenir une compensation fluctue, même dans le cas de poursuites semblables en apparence (reste-t-il quelque chose à saisir chez un débiteur?).

La judiciarisation des rapports sociaux, en matières civiles, est donc en bonne partie circonstancielle et soumise aux choix des justiciables. Ce qui ne signifie pas que les tribunaux jouent un rôle institutionnel mineur. Ils sont investis de pouvoirs exorbitants: déterminer ce qui a été, lorsque les faits sont contestés; dire ce qui en sera à l'avenir des créances et de la situation de tout un chacun. Qui plus est, eu égard à l'absence de régulation bureaucratique intensive propre aux sociétés contemporaines et au faible degré d'institutionnalisation des activités économiques, la Cour supérieure occupe certainement une place de choix, sinon le premier rang, parmi les instruments de régulation sociale durant la transition au capitalisme industriel. Le phénomène de judiciarisation des rapports sociaux, en ce sens, n'est pas l'apanage du temps présent. On peut même croire cette judiciarisation plus intensive à la fin du XIX ${ }^{\mathrm{e}}$ siècle, comme la cour régule alors des champs socio-économiques maintenant lourdement normés: consommation, transactions immobilières, prêts, affaires de famille, etc. Il en va de même en ce qui a trait à la juridicisation de la société. Certes, le volume de normes juridiques a littéralement explosé au cours du $\mathrm{XX}^{\mathrm{e}}$ siècle avec le développement des différentes branches de l’appareil d’État. Mais la juridicisation peut également être envisagée du point de vue de la présence ou proximité concrète du droit au sein des parcours des individus et organisations. Il est une mesure indirecte de la place qu'occupent la Cour supérieure et le droit civil dans la vie des justiciables du district de Trois-Rivières durant la décennie 1880. Il s'agit de la présence récurrente de certains plaideurs dans le contentieux de ce tribunal (tableau 4).

La mesure du phénomène est bien imparfaite et certainement inférieure à la réalité. Les poursuites entamées en dehors des trois années ciblées nous échappent. La graphie variable des patronymes et les risques d'homonymie entre individus différents (surtout du côté des francophones) ont fait en sorte qu'il a fallu jouer de prudence au moment de déterminer si le même justiciable était impliqué dans plus d'un litige ${ }^{79}$.

79. La comparaison des occupations s'est révélée utile, tout comme l'examen des lieux de résidence des parties et du contenu des déclarations. 
Tableau 4

Demandeurs et défendeurs récurrents

Cour supérieure, district de Trois-Rivières

Dossiers des matières civiles en général, I880- I885- 1890

\begin{tabular}{|c|c|c|}
\hline Nombre d'apparitions & Demandeurs & Défendeurs \\
\hline 2 & 92 & 88 \\
\hline 3 & 21 & 25 \\
\hline 4 & 10 & 11 \\
\hline 5 & 14 & 1 \\
\hline 6 & 3 & 2 \\
\hline 7 & 2 & 2 \\
\hline 8 & 1 & 2 \\
\hline 9 & 1 & - \\
\hline 10 et plus & $1^{\text {a }}$ & - \\
\hline
\end{tabular}

Source: BAnQ, CMCQ,TPII S3 SS2 SSSI, dossiers de I880, 1885 et 1890

a) II s'agit des 14 présences de la Banque d'Hochelaga en tant que demanderesse.

La plupart des plaideurs n'apparaissent qu'une seule fois dans les affaires portées à l'attention de la cour durant les années 1880,1885 et 1890 . C'est plus souvent le cas, proportionnellement, des défendeurs que des demandeurs. Nous avons d'abord considéré l'ensemble des occurrences de rôles de demandeurs (871) et de rôles de défendeurs (959), sans tenir compte des apparitions multiples des mêmes individus ou personnes morales. Ont ensuite été retranchées toutes les apparitions multiples de certains justiciables, d'un côté comme de l'autre ${ }^{80}$. Résultat, 451 demandeurs ne requièrent l'intervention du tribunal qu'à une seule occasion; 617 défendeurs ne sont visés qu'une seule fois par une réclamation. La Cour supérieure n'est pas un mécanisme d'arbitrage neutre. C'est une institution axée sur la poursuite.

La championne toutes catégories, côté poursuite, est la Banque d'Hochelaga. L'institution est impliquée dans 14 litiges qui tous, sauf un, concernent des billets promissoires dont le paiement se fait attendre. Pour le reste, deux types d'acteurs sociaux dominent parmi les justiciables qui s'adressent fréquemment à la cour. Ce sont les commerçants et les manieurs d'argent. Leur activité en justice est symptomatique d'une

80. Par exemple, dans le tableau 4, les 92 parties demanderesses qui apparaissent à deux reprises nous ont fait soustraire 184 occurrences de l'ensemble des rôles de demandeurs, et ainsi de suite. 
position de force dans une socio-économie régionale où le négoce et le crédit sont des phénomènes interindividuels, alors que les grandes corporations et les banques sont quasi absentes de ces secteurs.

Ce sont parfois uniquement des billets promissoires qui mènent au dépôt de poursuites par des marchands. La mécanique de ces effets de commerce, maintenant tombés en désuétude, mérite qu'on s'y attarde. Ces billets sont des «... reconnaissance[s] écrite[s] d'une dette, consistant dans une somme d'argent, avec la promesse absolue de la payer au créancier $[\ldots]$ pour valeur reçue ${ }^{81} »$. Le faiseur (ou souscripteur) du billet s'engage à payer la somme à l'ordre d'une autre personne, le preneur (ou bénéficiaire) du titre. Une échéance est stipulée: le billet est payable à demande ou à un moment précis. Le preneur peut endosser l'effet (il se retrouve dès lors endosseur) et transporter (céder) le billet à un tiers, qui prend alors le relais en tant que bénéficiaire. Une simple signature suffit pour endosser et transporter un billet. Fait important, il n'y pas de limite quant au nombre de transports dont un billet peut être l'objet et l'assentiment du faiseur ou des endosseurs précédents n'est pas requis pour effectuer ces transferts. Le faiseur et les endosseurs successifs sont aussi solidairement responsables du paiement de la somme stipulée envers le porteur ${ }^{82}$. La circulation de billets promissoires, au moyen de transports successifs, explique bien des poursuites visant simultanément de multiples défendeurs.

Leur usage est massif au XIX ${ }^{\mathrm{e}}$ siècle. Notre échantillon est composé à près de $30 \%$ de poursuites pour des billets promissoires impayés. Désiré Girouard, auteur d'un Essai sur les lettres de change et les billets promissoires paru en 1860, indique que ces billets constituent les principaux effets de commerce en circulation au Bas-Canada ${ }^{83}$. Les dossiers judiciaires ne font pas état, habituellement, des circonstances entourant la signature de billets promissoires et de la considération en vertu de laquelle ces obligations sont contractées. Il peut s'agir de sommes dues pour des transactions commerciales $^{84}$ ou de prêts ${ }^{85}$ dont le paiement ou le remboursement est différé. Malgré les silences des sources, le recours aux billets promissoires par les justiciables du district s'avère très significatif quant aux liens sociaux et

81. Désiré Girouard, Essai sur les lettres de change et les billets promissoires (Montréal, John Lovell, 1860), 5. 82. Ibid., 5, 61-62, 80, 93, 165-166 et 209-211.

83. Ibid., 209.

84. Des billets figurent notamment dans les actifs de marchands faillis. Le Journal des Trois-Rivières, 28 mars 1887 , p. 3, col. 2.

85. Des prêteurs sur billets s'annoncent dans la presse locale. Le Journal des Trois-Rivières, 30 décembre 1869 , p. 3, col. 3 . 
juridiques noués autour de l'argent (ou de la valeur, pour être plus précis) durant la transition au capitalisme industriel. Ce sont des promesses de payer et des effets interpersonnels. La socio-économie du district relève donc en bonne partie d'un enchevêtrement complexe de rapports juridiques de face-à-face et d'attentes échelonnées dans la durée.

Pierre Nolasque Chaillez règle ses comptes le 3 septembre 1890. Ce marchand de la paroisse de Saint-Stanislas entame cinq procès ce jour-là contre des habitants du même lieu. Six personnes sont visées: quatre cultivateurs, une veuve poursuivie conjointement avec l'un d'entre eux (probablement son fils) et un journalier. Dans tous les cas, des billets promissoires ont été signés à l'ordre de Chaillez et les jugements sont rendus en sa faveur, par défaut. C'est à n'en pas douter un homme qui compte, à l'échelle de sa paroisse. Il n'a jamais à répondre d'une poursuite, du moins durant les années échantillonnées ${ }^{86}$.

Charles Joseph Marchildon de Saint-Pierre-les-Becquets, désigné alternativement comme cultivateur et commerçant, commerçant ou marchand, constitue un bel exemple de pluriactivité. Il est demandeur à sept reprises, pour des objets variés: marchandises, contrat de livraison de bois, billets promissoires. Il requiert aussi une déclaration d'hypothèque sur une terre qui, à l'origine, garantissait un prêt de sa part mais qui a été vendue à deux reprises depuis. Un acte de transport lui a permis d'acquérir une créance née de la vente d'une terre, transaction dans laquelle il n'était pas impliqué. Marchildon n'apparaît qu'une seule fois parmi les défendeurs. Un manufacturier de chaussures de Montréal l'accuse de détenir illégalement un stock vendu à un tiers qui a fait faillite après la transaction. Marchildon rétorque qu'il a agi à la demande des curateurs à la faillite, défense qui ne trouve pas grâce aux yeux du juge ${ }^{87}$.

Chaillez et Marchildon appartiennent à un groupe social particulier, aux contours diffus : celui de notables influents à l'échelle locale et actifs sur le marché des biens, de l'argent et des créances. Le fait qu'ils se portent acquéreurs de créances, de temps à autre, pointe en direction d'une connaissance assez fine, de leur part, des transactions conclues dans leur communauté. Certes, ils n'ont pas l'envergure des magnats du bois dont

86. BAnQ, CMCQ, TP11 S3 SS2 SSS1, 1890, $\mathrm{n}^{\circ} 247$, Chaillez vs Thiffault; $1890, \mathrm{n}^{\circ} 248$, Chaillez vs Brouillet; 1890, $\mathrm{n}^{\circ} 249$, Chaillez vs Lafontaine; 1890, $\mathrm{n}^{\circ} 250$, Chaillez vs Perron; 1890, $\mathrm{n}^{\circ} 251$, Chaillez vs Perron.

87. BAnQ, CMCQ, TP11 S3 SS2 SSS1, 1885, $\mathrm{n}^{\circ} 87$, Marchildon vs Perreault; $1885, \mathrm{n}^{\circ}$ 149, Marchildon vs Marcotte; 1885, $\mathrm{n}^{\circ}$ 254, Marchildon vs Germain; 1885, $\mathrm{n}^{\circ}$ 255, Pelletier vs Marchildon; 1890, $\mathrm{n}^{\circ} 198$, Marchildon $v s$ Tousignant; 1890, $\mathrm{n}^{\circ}$ 207, Marchildon $v s$ Perreault; 1890, $\mathrm{n}^{\circ} 214$, Marchildon $v s$ Trudelle; $1890, \mathrm{n}^{\circ} 327$, Marchildon vs Potvin. 
les entreprises transforment le marché du travail à l'échelle du district et épuisent ses forêts. Mais du point de vue de leurs débiteurs, gens croisés au village, qui à l'église, qui dans un commerce, leur pouvoir est substantiel. La Cour supérieure joue certainement un rôle important dans la conduite de leurs affaires, lorsque vient le temps de concrétiser les profits escomptés.

S’ils pèsent moins lourd sur le contentieux de la Cour supérieure que certains marchands, quelques manieurs d'argent font tout de même preuve d'une activité judiciaire non négligeable. Ezekiel M. Hart se présente comme courtier et agent collecteur dans les trois poursuites qu'il entreprend en 1880 et en tant que bourgeois dans celle qu'il entame en 1885. Ses réclamations concernent: un acte d'obligation en vertu duquel un cultivateur a reconnu lui devoir $233,90 \$$, intérêts non compris; une substantielle créance immobilière (près de $6500 \$$ ) dont il a fait l'acquisition; une dette dont la nature n'est pas précisée; des sommes dues en vertu de deux actes d'obligation passés avec un avocat ${ }^{88}$.

La plupart des défendeurs assignés à plus d'une occasion se débattent au milieu de difficultés financières. Leur incapacité à solder des billets promissoires est centrale. L'année 1880 est difficile pour James Shortis, commerçant de Trois-Rivières. Il doit répondre successivement à sept poursuites pour dette. L'homme doit près de $6000 \$$ en capital, somme considérable. Plusieurs des réclamants font partie de l'élite, ce qui donne un aperçu des liens d'affaires de Shortis: un banquier (George Baillie Houliston), un grand entrepreneur forestier (Alexander Baptist) et un constructeur de chemin de fer de Québec (R. H. McGreevy) ${ }^{89}$. La chute de ce marchand de Trois-Rivières autrefois prospère est brutale ${ }^{90}$.

Les dossiers de billets promissoires impayés permettent de mettre au jour de véritables petits réseaux de faiseurs, preneurs et endosseurs de ces effets. Ces transactions en série semblent particulièrement fréquentes au sein d'une catégorie sociale particulière, celle des cultivateurs qui ne se contentent pas du travail de la terre et tentent leur chance sur le marché local des biens et des créances. C’est parfois risqué pour eux, mais aussi

88. BAnQ, CMCQ, TP11 S3 SS2 SSS1, 1880, $\mathrm{n}^{\circ} 31$, Hart vs Brunelle; 1880, $\mathrm{n}^{\circ}$ 103, Hart vs Renière ; 1880, n 156, Hart vs Brulé; 1885, nº 62, Hart vs Harnois.

89. BAnQ, CMCQ, TP11 S3 SS2 SSS1, 1880, $\mathrm{n}^{\circ} 7$, McDougall vs Shortis; 1880, $\mathrm{n}^{\circ} 38$, Houliston vs Shortis; $1880, \mathrm{n}^{\circ}$ 57, Vachon vs Shortis; $1880, \mathrm{n}^{\circ} 81$, Baptist vs Shortis; $1880, \mathrm{n}^{\circ} 87$, McGreevy vs Shortis; $1880, \mathrm{n}^{\circ} 104$, Banque Union du Bas-Canada vs Luckerhoff ; 1880, $\mathrm{n}^{\circ}$ 136, Druyer vs Shortis ; 1880, $\mathrm{n}^{\circ}$ 147, Vachon vs Shortis.

90. La base de données Mauricie: base de données en histoire régionale montre l'étendue des activités marchandes, immobilières et manufacturières de Shortis durant les années 1870. On assiste plutôt à la vente en justice de ses immeubles en 1881 et 1882 . 
pour les habitants du district mêlés à leurs entreprises d'une manière ou d'une autre.

Le cas de Narcisse Grenier est éloquent. Ce cultivateur et commerçant de la paroisse de Saint-Grégoire n'entame qu'une poursuite alors qu'il doit répondre cinq fois à des réclamations. Grenier poursuit en avril 1885 un certain Léon Arel, commerçant, pour 10000 \$. L’affaire ne paraît pas avoir cheminé au-delà d'une demande de bref d'assignation. Arel est-il tout simplement son débiteur? La réalité est plus complexe. François Rouleau, cultivateur, les poursuit de concert en mai suivant: Arel a signé un billet promissoire de 200 \$, payable à trois mois d'échéance, à l’ordre de Grenier. Ce dernier l'a transporté à Rouleau qui a fait de même auprès de la Banque Union du Bas-Canada. Le billet n’ayant pas été honoré, Rouleau a alors remboursé l'institution, intérêts compris. Il est redevenu par le fait même le bénéficiaire du titre et il actionne les deux hommes.

En octobre de la même année, tout s'emballe. La Banque d'Hochelaga poursuit Grenier, cette fois-ci en compagnie d'un autre cultivateur et commerçant, Ludger Turcotte. Le modus operandi est le même. Arel est à la source de deux billets (pour un total de près de 1720 \$) qui sont passés entre les mains de trois individus, dont Grenier, un autre Arel (Avila) et Turcotte, avant d'aboutir au comptoir de l'institution financière. Une autre poursuite entamée le même mois montre une distribution des rôles légèrement différente. Rouleau actionne conjointement Grenier et Turcotte. Grenier a signé un billet promissoire à l'ordre de Turcotte qui l'a ensuite transporté à Rouleau. Fin octobre, entre en scène un groupe d'héritiers, les Godin. Ils souhaitent que Grenier, Arel (Léon) et Turcotte fassent cession de leurs biens, comme ils détiennent à leur encontre une créance non garantie de plus de 8000 \$. Les mêmes héritiers reviennent à la charge en 1890. Ils s'en prennent cette fois à Grenier et à un médecin, Achille Fleury. Un billet signé par ce dernier à l'ordre de Grenier a abouti dans le patrimoine de Joseph Narcisse Godin, le testateur.

Toutes les poursuites relatives à des billets et visant ce petit réseau à géométrie variable se soldent par des condamnations. La procédure en cession de biens est suivie de saisies et de ventes d'immeubles ${ }^{91}$. Revenons-en à la première poursuite mentionnée, celle d'avril 1885. Grenier a-t-il tenté de mettre à l'abri une partie des avoirs de son partenaire Arel,

91. BAnQ, CMCQ, TP11 S3 SS2 SSS1, 1885, $\mathrm{n}^{\circ}$ 79, Godin vs Arel; 1885, n 106, Grenier vs Arel; 1885 , $\mathrm{n}^{\circ} 129$, Rouleau vs Arel; 1885, $\mathrm{n}^{\circ} 222$, Banque d’Hochelaga vs Grenier; 1885, $\mathrm{n}^{\circ} 235$, Rouleau vs Grenier; $1890, \mathrm{n}^{\circ} 163$, Godin vs Fleury. 
au moyen d'une poursuite simulée, avant que ne s'abattent sur eux les réclamations de tiers?

Des conflits familiaux impitoyables dégénèrent aussi en poursuites et contre-poursuites. Le dérèglement de la reproduction sociale des familles constitue une conjoncture proprement explosive au XIX ${ }^{\mathrm{e}}$ siècle. Les rapports interpersonnels, au sein des ménages et des lignées, sont indissociables des liens financiers et juridiques qui unissent les individus au patrimoine familial, du moins dans les milieux où l'on dispose d'un tel actif, si modeste soit-il. Si les poursuites à caractère économique renvoient à un capitalisme de face-à-face, il va sans dire que le caractère interpersonnel des obligations, droits et espérances est on ne peut plus net à l'échelle des familles. Tant l'espace public que la sphère privée sont ainsi marqués par une juridicisation intense.

Pamphile Pontiac Dutremblay connaît un parcours tortueux en justice. Cet arpenteur de Sainte-Anne-de-la-Pérade est deux fois demandeur et quatre fois défendeur durant la seule année 1885. En janvier, son épouse Marie Clémentine Dufort requiert une séparation de corps pour coups, injures et menaces de mort. À peu près au même moment, un rentier qui lui est probablement apparenté, Thomas Dutremblay, le ramène au tribunal: Pamphile lui doit une somme colossale, soit 11510 \$. Surprise, Pamphile confesse jugement - ce qu'il ne fait pas en d'autres temps - et se voit condamné à rembourser cette petite fortune le 2 avril 1885. Est-ce là une poursuite stratégique pour mettre à l'abri des capitaux que d'autres apparentés pourraient réclamer? Le même jour, Marie Anne Méthot fait opposition au jugement. Elle est issue du premier mariage de Marie Clémentine et Pamphile a déjà été son tuteur. La Cour de révision annulera, près d'un an plus tard, en 1886, le jugement du 2 avril 1885.

Marie Anne Méthot a aussi gagné une poursuite contre Pamphile en mars 1885 au sujet d'un billet promissoire. En septembre, l'épouse de ce dernier, Marie Clémentine, dont il est séparé de biens, réclame l'émission d'un bref de saisie. Elle allègue que Pamphile aurait déjà vendu à l'encan public une partie des biens lui appartenant. Notre homme contre-attaque par la suite. Il poursuit sa femme en novembre, alors qu'ils sont maintenant séparés de fait. Les deux époux avaient été condamnés, en tant qu’ex-tuteurs de Marie Anne, à verser un reliquat de compte de tutelle de près de $5600 \$$. Pamphile prétend qu'il n'avait pas à rembourser cette somme, au prétexte que c'est sa femme qui a profité des biens de la jeune fille. Il inclut deux tiers-saisis dans sa poursuite... Marie Anne et le mari de celle-ci. Le couple, dit-il, recèlerait des biens de sa femme. Marie Clémentine fait valoir plusieurs 
moyens de défense. Entre autres, elle aurait elle-même assumé les dépenses de leur ménage et payé certaines dettes de Pamphile, ce qui ferait de lui son débiteur. L’action est déboutée en avril 1886.

Changement de cible en décembre: Pamphile poursuit son ex-pupille Marie Anne pour près de 2700 \$ en provisions, pension et nourriture, ainsi qu'au motif que celle-ci aurait fait faire les foins et fait pacager des bêtes à corne sur deux de ses terres. La jeune femme, majeure depuis quelques mois seulement, soutient que ces terres lui appartiennent. Autre tentative d'échapper au reliquat de compte qu'il a été condamné à payer? Nouvel échec: l'action est déboutée en juin $1886^{92}$.

\section{CONCLUSION}

Les justiciables du district de Trois-Rivières impliqués dans les affaires entendues par la Cour supérieure durant les années 1880 ont un profil particulier. La juridiction de cette instance, chargée de voir aux réclamations les plus substantielles, y est pour beaucoup. Les cultivateurs sont sous-représentés parmi les demandeurs et défendeurs, alors que les commerçants forment la principale clientèle du tribunal, cela bien au-delà de leur poids réel dans la population active de la région. Ce sont les aléas des transactions conclues par les justiciables et les conflits divers qui ponctuent leur existence qui alimentent le contentieux de la cour. Celle-ci, par conséquent, ne peut être considérée comme un lieu où s'affrontent les intérêts divergents des classes sociales. Ses archives permettent toutefois de mettre en lumière des rapports de domination à l'échelle microsociologique, comme dans le cas de ces marchands et manieurs d'argent qui ont pour obligés et débiteurs plusieurs de leurs concitoyens. Il est une autre forme d'inégalité, instituée d'emblée par le droit, qui pèse très lourd sur l'identité des plaideurs: l'infériorité des femmes mariées. Il ne faudrait pas négliger, par contre, l'importance que put revêtir parfois pour elles la possibilité d'en appeler aux tribunaux civils pour mitiger, tant bien que mal, les effets structurels de leur situation désavantageuse.

Les décennies qui suivent la promulgation du Code civil du Bas-Canada constitueraient une phase particulière de l'histoire de la juridicisation et judiciarisation des rapports sociaux au Québec. Les rapports tissés autour des biens et de l'argent prennent la forme d'interactions et d'obligations interpersonnelles, de face-à-face. La manière dont les transactions sont

92. BAnQ, CMCQ, TP11 S3 SS2 SSS1, 1885, $\mathrm{n}^{\circ}$ 10, Dufort vs Dutremblay; 1885, $\mathrm{n}^{\circ} 72$, Dutremblay $v s$ Dutremblay; 1885, $\mathrm{n}^{\circ} 76$, Méthot vs Dutremblay; 1885, $\mathrm{n}^{\circ} 187$, Dufort vs Dutremblay; 1885, $\mathrm{n}^{\circ} 251$, Dutremblay vs Dufort; 1885, n² 260, Dutremblay vs Méthot. 
conclues, la façon dont naissent et circulent les créances et l'importance du patrimoine familial en ce qui a trait à la condition des personnes en témoignent. Les conséquences sont importantes du point de vue de l'étude de l'expérience du droit civil. Ce n'est pas tant la variété et la quantité de normes juridiques qui caractérisent cette époque, mais bien leur immédiateté, leur constante concrétude dans le parcours des justiciables. De même, l'arbitrage de la Cour supérieure et ses condamnations ont une importance significative. La faiblesse de l'institutionnalisation du marché - les institutions bancaires et les corporations industrielles sont très discrètes, du moins en justice - le recours massif au crédit et l'absence de bureaucratisation étatique des rapports sociaux font de ce tribunal une des pièces maîtresses, sinon la pierre d'assise, de la gestion et régulation de la société.

Âge d'or du droit civil, âge d'or des tribunaux? Il serait peut-être hasardeux d'en venir dès maintenant à une telle conclusion à partir du seul examen des plaideurs. Une analyse plus minutieuse de l'éventail et de la nature des litiges portés à l'attention de la cour s'impose. C'est ce à quoi nous consacrerons un second article qui viendra clore cette enquête. 